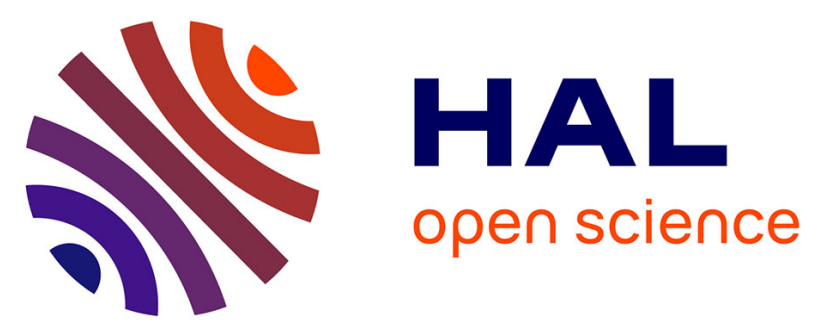

\title{
Le rôle des églises dans la colonisation portugaise du Gharb al-Andalus et dans l'organisation postérieure de l'habitat au sud du Tage (XIe-XV siècles)
}

Stéphane Boissellier

\section{- To cite this version:}

Stéphane Boissellier. Le rôle des églises dans la colonisation portugaise du Gharb al-Andalus et dans l'organisation postérieure de l'habitat au sud du Tage (XIe-XV siècles). XXXIIIe congrès de la SHMES, Société des historiens médiévistes de l'enseignement supérieur public May 2002, Madrid, Espagne. pp.115-131, 10.3406/shmes.2002.1831 . halshs-01460657

\section{HAL Id: halshs-01460657 \\ https://shs.hal.science/halshs-01460657}

Submitted on 7 Feb 2017

HAL is a multi-disciplinary open access archive for the deposit and dissemination of scientific research documents, whether they are published or not. The documents may come from teaching and research institutions in France or abroad, or from public or private research centers.
L'archive ouverte pluridisciplinaire HAL, est destinée au dépôt et à la diffusion de documents scientifiques de niveau recherche, publiés ou non, émanant des établissements d'enseignement et de recherche français ou étrangers, des laboratoires publics ou privés. 


\section{Le rôle des églises dans la colonisation portugaise du Gharb al-} Andalus et dans l'organisation postérieure de l'habitat au sud du Tage (Xle-XV siècles)

\section{Monsieur Stéphane Boissellier}

\section{Citer ce document / Cite this document :}

Boissellier Stéphane. Le rôle des églises dans la colonisation portugaise du Gharb al-Andalus et dans l'organisation postérieure de l'habitat au sud du Tage (Xle-XV siècles). In: Actes des congrès de la Société des historiens médiévistes de l'enseignement supérieur public, 33 ${ }^{\mathrm{e}}$ congrès, Madrid, 2002. L'expansion occidentale (Xle - XVe siècles) Formes et conséquences XXXIIle Congrès de la S.H.M.E.S. pp. 115-131;

doi : $10.3406 /$ shmes.2002.1831

http://www.persee.fr/doc/shmes_1261-9078_2003_act_33_1_1831

Document généré le 04/06/2016 


\title{
Le rôle des églises dans la colonisation portugaise du Gharb al-Andalus et dans l'organisation postérieure de l'habitat au sud du Tage (XII $-X V^{e}$ siècles)
}

\author{
Stéphane Boissellier
}

Les travaux organisés autour des concepts d'expansion territoriale et de colonisation prennent le plus souvent pour cadre de vastes espaces et pour objet des relations dans lesquelles les États interviennent lourdement, en liant d'ailleurs fortement la notion de colonisation à celle d'État'. C'est de réalités beaucoup plus locales et triviales, quoique en relation avec un vaste mouvement militaire (la "Reconquête ") au début de la période, que nous voudrions traiter, autour de la notion de "frontière intérieure ". En effet, quelles que soient les spécificités de l'expansion ibérique médiévale aux dépens d'al-Andalus, il est évident qu'elle constitue une colonisation, généralement analysée à travers le concept quelque peu réducteur de " repeuplement "; c'est dire que l'organisation de l'espace y constitue un phénomène majeur, fortement lié à des structures sociales et des conceptions politiques tout à fait originales. Mais cette organisation de l'espace peut être mise en rapport avec un phénomène plus global, la colonisation interne de l'Occident médiéval, c'est-à-dire l'humanisation d'espaces jusqu'alors vides ou la réorganisation d'espaces moins densément occupés (notamment à travers l'incastellamento). Les deux formes, colonisation et humanisation, qui correspondent grosso modo à la distinction traditionnelle entre colonies d'encadrement et colonies de peuplement, se superposent dans la zone que j'étudie ; socialement, les deux formes, diaspora aristocratique et migration

1. Voir par exemple les diverses contributions à Élat et colonisation au Moyen Âge et à la Renaissance, M. BALARD dir., Lyon, 1989. 
paysanne, se confondent dans l'institution municipale du Midi portugais ; en effet, l'immigration de chevaliers roturiers dans des castra coloniaux, qui, en tant que "chefs-lieux", organisent l'espace alentour pour le compte du seigneur, met en place des communautés auxquelles des chartes municipales confèrent ou reconnaissent une dynamique de domination: colonies de peuplement, les élites des chefs-lieux constituent aussi des pôles « seigneuriaux » d'organisation, comme les villes franques de Terre sainte.

Dans ce double mouvement, les églises (en tant que bâtiments concentrant des fonctions) jouent un rôle que l'on sous-estime par rapport à d'autres éléments matériels, comme les fortifications ou les terroirs. D'une part, elles jouent un rôle idéologique, christianisant l'espace par leur seule existence ; c'est une modalité que l'on peut entrevoir à travers les titulatures célestes et la localisation des lieux de culte (notamment les chapelles sans habitat associé) : l'aspect le mieux étudié de ce problème mais qui n'est peut-être pas le plus décisif est la christianisation des mosquées. D'autre part, d'un point de vue social, les lieux de culte, quel que soit leur statut cultuel, sont des instruments d'organisation des communautés de colons et de polarisation de l'espace à toutes les échelles. Les petits habitats qui se constituent dans la périphérie des chefs-lieux castraux sont parfois agglomérés autour d'une chapelle; que celle-ci soit antérieure à l'habitat (constituant un bourg ecclésial) ou contemporaine, elle joue un rôle d'identification locale majeur que l'on retrouve dans le toponyme (qui est parfois un hagiotoponyme). Largement intégrée dans le pouvoir de ban, l'église est soumise au patronage des seigneurs publics; à ce titre, l'église appuie la politique des seigneurs qui dirigent la colonisation, notamment quand elle est chef-lieu de paroisse. Elle assoit et organise la plus ancienne fiscalité banale réelle (les dîmes prédiales), dans une région où les prélèvements sont très majoritairement personnels durant la Reconquête.

\section{La constitution d'un réseau épiscopal}

Avant d'être un instrument d'identité locale et/ou d'administration seigneuriale, le lieu de culte (à l'exception des chapelles privées) est l'élément d'un ensemble, le réseau ecclésiastique, et sa place dans cet ensemble conditionne et révèle le statut de l'habitat qui l'abrite. Jusqu'à ce que les habitats s'intègrent dans une hiérarchie civile claire avec une titulature fixe, c'est l'église qui est le principal indice de la centralité des habitats ; en effet, les lieux de culte, contrairement aux habitats en tant que tels, ont un véritable statut relatif qui les intègre dans une hiérarchie théoriquement rigoureuse (s'exprimant par des vocables uniformisés) ; or, cette hiérarchie est imposée par le haut et c'est une volonté centralisatrice et régulatrice, celle de l'évêque, qui informe de plus en plus ces rapports ${ }^{2}$. En

2. Même avant 1200-1220, quand les évêques semblent absents de l'organisation ecclésiastique, le droit leur confère des prérogatives sacrales exclusives sur les fidèles, les 
outre, par-delà le flou de la définition des chefs-lieux paroissiaux par leurs fonctions, la paroissialité se définit par un critère essentiel, le lien administratif entre l'église locale et la capitale épiscopale, à travers la délégation de la cura animarum.

La Reconquête - qui imprime, rappelons-le, toute la civilisation ibérique depuis le VIII ${ }^{\mathrm{e}}$ siècle - engendre une structure spatiale (à l'échelle régionale) latitudinale, à laquelle s'adapte imparfaitement l'organisation diocésaine qui l'accompagne et qui se veut, elle, la véritable " restauration " d'une organisation fondée sur des facteurs tardo-romains : si, au sud du Tage, l'adéquation est assez notable entre diocèses restaurés et structures socio-culturelles, la réorganisation diocésaine "longitudinale " entre Mondego et Tage s'oppose à la logique de front pionnier (du nord vers le sud) - ce qui constitue d'ailleurs un paradoxe car les diocèses et les provinces les encadrant, à l'époque wisigothique, semblaient s'organiser eux aussi en latitude. On ne peut malheureusement que supposer la forme des diocèses paléochrétiens car, à l'exception de celui de Braga - connu par le Parochiale suevicum -, on ne connaît que leur siège et non leurs contours ; en revanche, les rapports spatiaux entre sièges permettent d'affirmer que les provinces ecclésiastiques de l'Occident ibérique sont nettement latitudinales. En outre, malgré le "fondamentalisme wisigothique » des restaurateurs, les considérations nationales se sont souvent imposées durant la Reconquête : si les provinces ecclésiastiques antérieures à l'invasion arabo-musulmane découpaient le Portugal de part et d'autre du Douro (diocèses suffragants de Braga au nord, de Mérida au sud), la Reconquête établit une ligne de partage oscillant entre Mondego et Tage.

Notre région dépend de quatre sièges (Lisbonne, Idanha, Évora et Silves) qui sont tous des évêchés wisigothiques " restaurés ", parfois au prix d'un léger déplacement (Silves à la place de l'antique Ocsonoba, Idanha finalement installé à Guarda); mais c'est le siège d'Évora (restauré en 1166) qui joue le rôle le plus important puisqu'il « repousse " l'influence des autres sièges vers le nord et empêche - fait rarissime - la restauration du siège wisigothique de $\mathrm{Beja}^{3}$. Ces diocèses constituent dans le sud, pour les

temples et les clercs, prérogatives qu'ils ne peuvent manquer d'exercer : ainsi la définition (tout à fait canonique) de la juridiction épiscopale dans un accord de "réception " de l'évêque d'Évora dans les églises d'Arronches par le seigneur et patron du lieu, le monastère Sainte-Croix de Coimbra en 1248 (Arquivo Nacional da Torre do Tombo, S. Cruz de Coimbra, maço 18, doc. 40). Dans cette optique, les chefs-lieux paroissiaux ne sont que des relais de l'évêché et la construction diocésaine est la réalité première; la législation

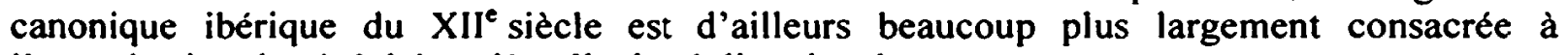
l'organisation des évêchés qu'à celle des églises locales.

3. Peut-être parce que le titre de ce dernier, diocesis pacensis, a été "transféré ", dans des conditions obscures, à un autre évêché unique en son genre (de fondation mozarabe, au IX $\mathrm{X}^{\mathrm{e}}$ siècle), Badajoz (cf. A. LAMBERT, art. "Badajoz " dans Dictionnaire d'histoire et de géographie ecclésiastiques, Paris, 1932, t. VI, col. 100-108); l'hypothèse d'un oubli de l'épiscopalité pré-musulmane de Beja au XIII siècle est peu crédible (J. PÉrEZ DE URBEL, art. "Beja » dans ibid., t. VII, col. 481-484). La fiction de la continuité wisigothique pèse d'un tel 
organisateurs du peuplement et des pouvoirs, le seul cadre administratif dont le souvenir s'est conservé à travers l'Islam et dont l'établissement est le plus facile à légitimer car sous forme de rétablissement d'une réalité préexistante ; contrairement aux évêchés du centre et du nord, les sièges épiscopaux méridionaux, à partir de la prise de Lisbonne, sont rétablis dès la conquête du lieu (sauf Idanha), ce qui montre qu'ils s'intègrent désormais dans la panoplie des instruments de la colonisation ${ }^{4}$. Le rôle primordial de la monarchie dans l'aménagement des régions méridionales n'est évidemment pas étranger à cette utilisation des sièges épiscopaux ; il est clair que, avant la fin du XIII ${ }^{e}$ siècle, les évêques méridionaux sont à leur façon des agents de la toute première "centralisation " monarchique, jusqu'à ce que celle-ci finisse par heurter l'appareil ecclésiastique, qui s'est construit et a acquis sa propre dynamique entre-temps ${ }^{5}$. Il faut noter par ailleurs que les évêques ayant juridiction au sud du Tage ne détiennent pas (ou pas longtemps) de seigneuries politiques territoriales ${ }^{6}$, notamment dans la ville qui est siège de leur diocèse (contrairement aux évêques « féodaux » septentrionaux de Porto et Braga); ils construisent donc leur diocèse principalement comme une sphère juridictionnelle détachée du pouvoir direct sur les hommes et non pas comme une seigneurie concurrente.

Juridiquement, la priorité de l'action épiscopale et la légalité de ses instruments sont indéniables mais les droits du clergé sur des zones en cours d'occupation sont loin d'être clairs' et sont récusés non seulement par les chefs du peuplement mais aussi par les communautés d'habitants ellesmêmes ${ }^{8}$. En outre, la restauration d'un diocèse est une opération complexe,

poids dans les structures ecclésiastiques qu'on élabore la fiction d'un transfert du siège de Beja à Badajoz pour légitimer son antiquité.

4. Restaurations des évêchés de Lisbonne en 1147, Évora en 1166 et Silves en 1189.

5. H. VILAR, As dimensões de um poder. A diocese de Évora na Idade Média, Lisbonne, 1999, p. 27-95.

6. L'évêque d'Évora exerce le pouvoir banal sur les villages de Vimieiro, Arraiolos et Alcáçovas mais seulement jusqu'en 1271 ; son pouvoir sur quelques hameaux en seigneurie foncière dans les alentours d'Évora est plus durable mais ils sont inclus dans la juridiction du chef-lieu et le pouvoir exercé sur les hommes n'est pas de nature publique. L'évêque d'Idanha octroie une charte à Alter do Chão.

7. En 1237 , l'évêque d'Idanha obtient du pape une charte lui octroyant le droit d'annexer à son diocèse des lieux déserts et castra abandonnés, sans forcément en assurer le repeuplement (Monumenta Henricina, Coimbra, 1960, t. I, p. 62-63); on peut se demander s'il s'agit d'un moyen d'étendre les limites de son diocèse (en englobant des lieux qui ne dépendent encore d'aucun siège épiscopal) ou de lui garantir préventivement une juridiction partout où il n'y a pas encore d'église. Le diplôme est d'ailleurs prudemment octroyé sine prejudicio juris alieni.

8. L'opposition des seigneurs aux prérogatives épiscopales n'a pas besoin d'être soulignée puisque tous les accords avec les évêques en portent la trace; pour l'opposition communautaire, on possède une bulle très intéressante de 1216 qui révèle que les habitants de lieux frontaliers, profitant de la rupture des situations légales induite par les offensives almohades des années 1170-1190, refusent l'autorité épiscopale loca posita inter te ac eosdem [infideles] a fidelibus populantur qui te volente in illis exercere pontificale officium et jura episcopalia exigente maliciose negant loca ipsa esse de diocesi Elborensi, affirmantes 
qui se fait aux dépens d'autres ayant-droits. Dans le centre du Portugal, certains évêchés (Coimbra, Lamego, Viseu, Idanha) ne sont " restaurés " que longtemps après la conquête de la région qu'ils dirigeaient à l'époque wisigothique et ils ont à faire alors à des églises locales plus anciennes que l'église siège et ayant donc été gouvernées depuis un autre centre épiscopal, plus lointain (Coimbra et Porto depuis Braga). De la même façon, les églises de toute la zone entre Lisbonne et Évora conquise avant 1166 sont rattachées au siège de Lisbonne (comme l'importante église Sainte-Marie d'Alcácer, fondée dès la première conquête chrétienne en 1158 ou 1160$)^{9}$. Mais la destruction, lors de la contre-offensive almohade des années 1170-1190, de presque toutes ces églises méridionales, sauf précisément la ville siège du diocèse (Évora), facilite la prise en main des églises par le siège lors de la récupération définitive du Ribatejo et du Haut Alentejo à partir de 1190.

Si la plupart des églises rattachées au siège sont donc de fondation (définitive) postérieure à celui-ci dans le diocèse d'Évora, beaucoup d'entre elles ont été fondées en dehors de tout contrôle épiscopal. En fait, dans ce diocèse, l'emprise épiscopale sur les fondations est très faible jusqu'aux années 1220 et limitée jusqu'aux années 1270 , c'est-à-dire durant la grande phase de mise en place des temples. Cependant, l'action épiscopale, malgré les résistances, prépare l'avenir. Par l'ordination des prêtres, les évêques disciplinent l'exercice du droit de patronage ; par la consécration des autels et l'octroi du titre paroissial, ils constituent un réseau à l'échelle de toute la région (au moins dans le cadre du diocèse d'Évora), sans effets directs sur la répartition du peuplement mais qui prépare l'administration territoriale monarchique du Bas Moyen Âge. À défaut d'en créer la trame, l'évêque hiérarchise les habitats; il peut donc y avoir inéquation entre les fonctions locales (et supra-locales) d'une église et son insertion dans le réseau hiérarchique diocésain.

Dans un contexte de colonisation, le cadre diocésain se construit en même temps que les autres structures d'organisation de la société ; les sièges épiscopaux restent longtemps des têtes quasiment sans corps, au moins pour les évêchés d'Évora et Silves. Contrairement aux diocèses créés par redécoupage dans les zones de vieille christianisation, ceux des régions coloniales partent de rien et l'évêque semble perçu non pas comme le chef d'un territoire mais comme le chef d'une simple collégiale locale émettant des prétentions exorbitantes à s'emparer dans toute la région d'une structure

eadem spectare ad aliquam cathedralium sedium que ab infidelibus detinentur (Bulário português. Inocêncio III (1198-1216), A. J. DA COSTA et M. A. F. MARQUES éd., Lisbonne, 1989 , p. 390).

9. En témoigne la chronique du siège de Lisbonne : sedes episcopatus cum hiis castris et vicis subscriptis, trans Tagum castro Alcacer, castro de Palmella, Elmada provincia [...] Sunt autem termini eius ab Alcacer castro usque ad castrum Lora [Leiria] et a mari occidentali usque civitatem Eburensem (A conquista de Lisboa aos mouros. Relato de um Cruzado, A. A. NASCIMENTO éd. et trad., Lisbonne, 2001, p. 142). 
qu'il n'a pas créée ${ }^{10}$. Cette faiblesse originelle confère en revanche une potentialité immense à l'action épiscopale; sans constituer un cadre déterminé dès l'origine, le diocèse est construit à partir d'un seul centre, donc dans un processus de polarisation parfait, et sa construction administrative suit une progression géométrique : la fixation, généralement conflictuelle, des frontières entre diocèses est donc particulièrement révélatrice du processus de construction des espaces administratifs puisqu'elle résulte de la "rencontre » entre des zones d'influence. Les évêques constituent plus ou moins rapidement la seule autorité ayant une vue et un plan d'ensemble au niveau régional. Cette planification de l'espace - à laquelle on ne peut comparer que celle qui est menée par les monarques en gérant les fortifications et en distribuant les juridictions - s'exerce dans un sens d'autant plus clair que la hiérarchie des lieux de culte est de droit.

Mais du point de vue spatial, aussi bien au niveau local qu'à l'échelle du réseau tout entier, la paroisse constitue une instance administrative supralocale qui reste abstraite. En effet, ne peuvent être qualifiés de chefs-lieux "paroissiaux »-que les documents désignent d'ailleurs rarement comme tels - que les églises directement liées à l'administration épiscopale; ce ne sont pas la territorialité ni les fonctions (centralisation et répartition des dîmes et monopole de sacrements) qui définissent dans notre région la paroissialité mais la reconnaissance de l'évêque comme autorité de tutelle par le patron, l'institution directe (sous forme de confirmation) du desservant par l'évêque (i.e. délégation de la cura animarum) ${ }^{11}$, l'exercice par celui-ci du droit de visite (avec perception de la "procuration " ou collecta) ${ }^{12}$ et

10. Dans les faits, le rétablissement d'un siège épiscopal ne signifie pas le rétablissement du territoire diocésain, ne serait-ce qu'à cause de l'ignorance des limites diocésaines wisigothiques (sauf celles suggérées dans le nord par le parochiale suevicum) lors de la Reconquête ; les conflits entre diocèses pour la fixation de leurs frontières témoignent de cette ignorance. En 1189, la donation du castellum d'Alvor aux chanoines de Sainte-Croix de Coimbra présente l'évêque du tout nouveau dioècese de Silves comme eiusdem provintie et regionis [Silvii] primo tunc temporis pontifice (Documentos de D. Sancho I (1174-1211), P. Rui De Azevedo, A. de J. Da Costa et M. Rodrigues Pereira éd., Coimbra, 1979, p. 64); comme le eiusdem renvoie à Silves en tant que chef-lieu du village d'Alvor, le diocèse est plutôt un point, éventuellement central, qu'un cadre.

11. Cet élément est un facteur essentiel de la « centralité » des églises paroissiales par rapport à celles qui ne le sont pas et qui leur sont soumises; les actes ne sont pas toujours clairs à ce sujet, réglant souvent la présentation/confirmation « des clercs " sans précision, mais l'accord de 1236 entre l'ordre d'Avis et l'évêque d'Évora au sujet des églises de Seda et Fronteira, distinguant nettement les églises paroissiales des deux chefs-lieux et les autres églises (de qualibet ecclesia parrochiali in predictis villis sita), montre clairement que l'évêque n'institue que les recteurs des églises-mères (statutum est quod ydoneus frater quem magister et fratres presentaverint, episcopus recipiat et acceptet in qualibet ecclesia parrochiali), C. DA Silva TAROUCA, "As origens da Ordem dos cavaleiros de Évora (Avis) segundo as cartas do Arquivo do Cabido da Sé de Évora ", A cidade de Évora, 5 (1947), p. 25-39, ici p. 37.

12. Dès les plus anciens accords entre seigneurs banaux et évêques d'Évora et Idanha, le montant de la collecta est fixé (par exemple, vers 1217 , Ibid., p. 34) et sa seule mention atteste que la "réception » de l'évêque institue une juridiction directe de celui-ci, désormais autant au temporel qu'au spirituel. 
l'affectation d'une part canonique des revenus ecclésiastiques - et c'est ce dernier caractère qui justifie le versement à l'église " épiscopale » des dîmes perçues par les chapelles locales. Outre l'adjectif parrochialis qualifiant çà et là des églises, on a une image globale fiable, mais seulement tardive, du réseau épiscopal avec la liste (en 1371) des églises soumises directement au paiement des procurations à l'évêque (i.e. soumises à la visite paroissiale). À cette date, ce sont quelque 90 églises du (seul) diocèse d'Évora qui sont sûrement soumises au pouvoir direct de l'évêque ${ }^{13}$ et leur répartition se conforme étroitement à celle des chefs-lieux politiques parce que les évêques ont privilégié le contrôle direct des églises les plus anciennes, les plus riches et exerçant une juridiction sur d'autres temples.

\section{L'église comme instrument d'administration et facteur de hiérarchie}

Les lieux de culte servent d'instrument d'organisation de la population. En effet, l'accumulation de grandes églises paroissiales sur un même lieu (les chefs-lieux municipaux anciens), particulièrement en Haut Alentejo, aux dépens de périphéries durablement dépourvues d'églises baptismales, au mépris d'une bonne administration territoriale, ne peut répondre uniquement aux nécessités spirituelles des assez faibles groupes de chrétiens qui y résident; ces églises sont avant tout des instruments de canalisation des dîmes au profit du chef-lieu et c'est au chef-lieu qu'il est le plus facile de capter les revenus car c'est là que résident les détenteurs des domaines agricoles les plus importants, les chevaliers vilains. En outre, les périphéries peuvent être négligées car la population chrétienne y est en faible nombre dans les premières décennies de la colonisation et les chrétiens qui y résident, assez infériorisés, ne sont pas encore en situation de réclamer l'autonomie de leur chapelle.

Durant la Reconquête, avec une administration seigneuriale embryonnaire, pesant sur des groupes de colons mal contrôlables, l'église constitue un instrument légitime et efficace de la collecte des prélèvements, qui sont le principal signe de soumission au pouvoir (autant et plus qu'une source de revenus). Dans un système politique où le pouvoir du seigneur s'exerce essentiellement sur la population du chef-lieu ayant reçu une charte, le contrôle sur les hommes du terminum est seulement indirect (à travers les élites dirigeantes du chef-lieu); le principal agent représentant directement le seigneur dans les bourgades est donc le prêtre. Ainsi, l'ordre de Santiago articule étroitement ses commanderies avec les églises centrales dont il est

13. Quinze églises sont exclues de ce décompte pour n'avoir rien payé ; ce peut être tout simplement par impécuniosité mais on sait que la liste prend pour matrice le rôle de décime de 1320 qui inclut des églises non épiscopales et cela peut expliquer la présence d'églises qui ne sont suivies d'aucune mention de somme parce que non soumises à procuration (Archivio Segreto Vaticano, Camera apostolica, Collectoriae 179, fol. 76-80). 
fondateur et patron puisque la commanderie est parfois conçue comme le temporel de l'église ${ }^{4}$. C'est dans la juridiction de l'ordre d'Avis, qui a construit l'essentiel de son réseau ecclésiastique directeur avant l'intégration diocésaine (en 1279), que l'on observe le mieux le lien entre hiérarchie des lieux et fonctions des églises, à travers les relations entre les "églisesmères » des chefs-lieux et leurs " chapelles " dans les villages politiquement dépendants. Bien sûr, les seigneurs détenteurs de plusieurs églises, même les plus volontaristes, semblent adapter la hiérarchie religieuse aux évolutions du peuplement, ce qui n'empêche pas une certaine part d'arbitraire dans les décisions ${ }^{15}$. Ce qui prouve l'usage des églises comme instrument de gouvernement, c'est la grande adéquation entre la carte des juridictions banales et celle des patronages (soit que le seigneur soit directement fondateur des églises, soit qu'il réussisse à s'emparer du patronage).

C'est essentiellement la capacité de prélèvement des églises, depuis la généralisation de la dîme, qui constitue un élément d'administration publique. En effet, le prélèvement seigneurial porte très peu sur les activités productives agricoles dans la région car il est plus facile de justifier le prélèvement sur les activités exercées directement par les élites des chefslieux, guerre et commerce; cependant, les seigneurs ne peuvent renoncer aux revenus que confère la ponction sur les cultures et le droit de patronage sur les églises leur permet de s'octroyer au minimum (pour les églises " épiscopales ") un tiers des dîmes et autres revenus canoniques, qui pèsent, eux, sur la totalité des activités ${ }^{16}$. À partir des années 1250-1280 (selon les

14. La commanderie d'Ourique est constituée par la comendam ambarum seu temporale des deux églises locales Sainte-Marie et Saint-Sauveur (Arquivo Nacional da Torre do Tombo, Santa Cruz de Coimbra, livro 97, $\mathfrak{f}^{\circ} 39 \mathrm{v}$ ). Dans les autres Ordres, ce phénomène se restreint aux grandes villes dans lesquelles ils n'ont pas d'emprise politique et foncière importante et où la commanderie est fondée principalement sur le patronage d'une église (ainsi, celle de l'ordre d'Avis à Elvas, fondée sur les revenus de l'église Sainte-Marie da Alcáçova ; à Lisbonne, la commanderie de l'ordre de l'Hôpital est la bailia de Saint-Bras. Cf. l'épitaphe du commandeur en 1346, M. J. BARROCA éd., Epigrafia medieval portuguesa (862-1422), t. II : Corpus epigráfico medieval português, Lisbonne, 2000, p. 1671.

15. Dans l'accord de 1279, le rapport entre les églises de Fronteira et Vide ne va pas de soi, peut-être parce que les deux lieux sont de fondation contemporaine et de contenu matériel et juridictionnel equivalent (aucun n'ayant de charte municipale), et il faut donc décider d'un lien subordonnant la seconde à la première (quam ecclesiam Sancte Marie de Vite statuimus esse capellam dicte ecclesie Sancte Marie de Fronteira, Arquivo Nacional da Torre do Tombo, Ordem de Avis, maço 2, doc. 129); cela peut correspondre à une organisation prenant Avis comme centre géométrique de référence et soumettant donc le lieu le plus extérieur (et donc le plus voisin de juridictions concurrentes comme Alter do Chão) au lieu le plus intérieur.

16. Les plus anciennes bulles pontificales, La documentación pontificia hasta Inocencio III (965-1216), D. Mansilla Reoyo éd., Rome, 1955 (Monumenta Hispaniae Vaticana. Sección registros, vol. I) ; Papsturkunden in Portugal, C. ERDMANN éd., Berlin, 1927 (Abhandlungen der Gesellschaft der Wissenschaften zu Göttingen. Philologische-historische Klasse, Ncuc Folge, XX, 3) et Bulário português, op. cit., attestent largement que les dîmes constituent l'enjeu financier majeur entre les diverses autorités colonisatrices, particulièrement les ordres militaires (par exemple, en 1179-1 181, Papsturkunden, op. cit., p. 254-255 et 262-263). 
zones), l'agriculture devient l'activité majeure et l'on observe que les dîmes constituent alors la principale source de revenus des seigneurs banaux. Outre le profit, l'intérêt de l'opération est d'exercer un contrôle de la totalité des activités, servant plus tard à asseoir de nouvelles exigences dans la mesure où les activités agricoles, dans la région, se développent en se fondant sur une main d'œuvre infériorisée qui échappe largement au contrôle du seigneur public : à travers le contrôle indirect des revenus issus de cette activité, les seigneurs préparent la prise en main de la totalité des sujets. De plus, c'est à travers le lien fiscal réel que se développe la territorialité ; l'église est donc l'instrument indispensable à l'assise de liens politiques manquant de matérialité ${ }^{17}$. Plus largement, la présence d'un lieu de culte sur un site colonisé est un élément même de la colonisation, par sa valeur symbolique ("restauration» de la présence divine en terre impie) et mercantile (comme service offert aux colons pour les attirer).

L'église est aussi un facteur de hiérarchie, dans la mesure où certains habitats dépourvus de lieu de culte dépendent d'autres pour la totalité de leur vie religieuse et dans la mesure où les lieux de culte ne détiennent pas tous les mêmes prérogatives cultuelles et financières. Un cas tardif bien détaillé nous permet d'aborder l'application administrative des différences de statuts. En 1437, le roi Duarte donne son droit de patronage sur l'église paroissiale Saint-Jean de Cartaxo à une autre église paroissiale, limitrophe, Sainte-Marie d'Aveiras (pour compenser à celle-ci la perte de revenus qu'elle a subie par la rétention d'une chapelle de son territoire, Sainte-Marie des Vertus, pour y fonder un monastère franciscain); le roi demande en conséquence à l'archevêque de Lisbonne d' "incorporer" l'église de Cartaxo à celle d'Aveiras, ce qui a pour effet de transférer au recteur d'Aveiras l'utilisation des revenus de l'église de Cartaxo et de conférer au même recteur la nomination du desservant de Cartaxo ${ }^{18}$. Juridiquement, la première église devient filiale de la seconde puisqu'elle perd son autonomie financière : le recteur devient salarié et non plus gestionnaire. Mais quand il s'agit de déterminer les implications de cette relation hiérarchique dans la centralité des lieux de culte concernés (collecte de la dîme et prérogatives cultuelles), les choses se compliquent singulièrement. Ainsi, il n'est pas dit que la perception des revenus (c'est-à-dire le paiement par les fidèles) soit effectuée par le recteur de l'église "chef-lieu " et, de fait, les listes de bénéfices ecclésiastiques mentionnent de nombreuses églises par lesquelles transitent

17. La fondation dans un modeste castrum comme Alenquer d'une collégiale (Saint-Étienne) de quinze prébendiers: Monumenta Portugaliae Vaticana III-2. Súplicas do pontificado de Martinho $V$ (anos 1-7). Documentos publicados com introdução e notas, A. DOMINGUES DE SousA COSTA, éd., Braga-Porto, 1982, p. 521, est manifestement un moyen d'organiser la fiscalité.

18. Que qualquer que por os tempos for prior da dicta igreja d Aveiras possa cometer $e$ deputar todos os fructos novos, rendas e dizimas e direitos da dicta igreja do Cartaxo em prol e husos da dicta igreja sua d'Aveiras. E que el dicto prior d'Aveiras e todos seus sucesores ponham em a dicta igreja do Cartaxo capellam (Chancelarias portuguesas. D. Duarte (1433-1438), J. J. Alves Dias éd., Lisbonne, 1998, vol. I-2, p. 100). 
fatalement des revenus alors que leur "pleine " paroissialité (i.e. contrôle épiscopal) est improbable.

Quoique moins détaillés, les plus anciens documents montrent bien qu'un rôle social et administratif différent est dévolu aux lieux de culte en fonction de leur statut : vers 1200 , quand est fixé (contentieusement) le statut de la chapelle Saint-Michel d'une auberge fondée par le futur ordre d'Avis dans son siège de l'alcáçova d'Évora, cette église se voit refuser la collecte des prélèvements canoniques, l'usage des cloches et la desserte des missas publicas (sauf pour le jour de la Saint-Michel, patron céleste du lieu) ${ }^{19}$; la réduction du lieu de culte à des fonctions strictement privées (incluant la sépulture et les oblations volontaires) est claire. Ce sont à peu près les mêmes restrictions qui affectent, lors d'un règlement contentieux en 1207, une chapelle de l'ordre de Santiago à Arruda (lieu dont l'Ordre est seigneur) dans le but évident de préserver les attributions et les revenus de la matrix ecclesia Sainte-Marie, qui est chef-lieu de la paroisse dans laquelle la chapelle semble être incluse ${ }^{20}$. Mais ces documents sont difficiles à interpréter en termes d'organisation ecclésiastique et surtout de rapports entre habitats. On est tenté d'y voir une clarification des statuts des lieux de culte (pour promouvoir la paroisse comme cellule d'encadrement, selon les directives grégoriennes) mais le conflit d'intérêts entre patrons semble prioritaire - les deux églises-mères étant respectivement sous le patronage de l'évêque d'Évora et du monastère canonial Saint-Vincent de Lisbonne. En outre, dans le cas d'Arruda, on ignore si la chapelle se localise physiquement dans le village d'Arruda ou dessert un habitat mineur distinct : dans le premier cas, l'inclusion territoriale dans une paroisse justifie l'infériorité tandis que, dans le second cas, il peut y avoir concurrence entre le chef-lieu et les habitats qu'il prétend dominer ${ }^{21}$.

La tendance à créer des collégiales (et peut-être à gonfler leurs effectifs), qui concentrent les revenus et les services religieux dans les chefslieux, constitue un frein important à la multiplication des temples publics locaux. En effet, ce sont l'entretien du bâtiment et surtout le salaire du chapelain qui posent le plus de problèmes financiers aux groupes candidats à l'autonomie religieuse car il y a toujours une phase durant laquelle le prieur ou le collège de l'église décimale résiste à reverser une part de son tiers des dîmes aux " filles " qui sont en train de lui naître, bien que ces dîmes soient normalement en augmentation - puisque c'est la croissance démographique des noyaux d'habitats qui justifie l'acquisition d'un lieu de culte propre; les fidèles des nouveaux habitats (noyaux ou zones) doivent donc financer leur lieu de culte en plus du paiement de la dîme au chef-lieu de paroisse. La résistance aux dîmes parfois signalée ${ }^{22}$ est généralement interprétée par les

19. C. Da Silva Tarouca, op. cit., p. 30-31.

20. Bulário português, op. cit., p. 254-255.

21. En effet, à cette époque, la définition de la zone de juridiction des églises-mères au-delà du tissu aggloméré fait souvent défaut.

22. Les visitations et les statuts synodaux dénoncent particulièrement ce défaut. 
autorités comme l'impiété de " mauvais riches " mais elle peut très bien une correction spontanée de l'injuste redistribution entre les différents lieux de culte.

Si l'on perçoit assez bien la volonté des «villageois " d'acquérir leur autonomie religieuse, spontanément ou à l'instigation d'un seigneur noble donataire, il est plus difficile de déterminer si les dirigeants populaires des chefs-lieux soutiennent la résistance de leur église paroissiale à cette autonomie, de la même manière qu'ils s'opposent directement aux démembrements de leur juridiction civile; l'église capitale peut être considérée par les habitants du chef-lieu à la fois comme un instrument de domination des habitants du termo et comme le cadre de leur propre oppression par les seigneurs (politique et religieux) ${ }^{23}$. Il est probable que c'est un clivage social qui partage les deux opinions au sein des « citadins».

\section{L'église, instrument d'identité}

Entre le milieu du XII ${ }^{e}$ siècle et la fin du Moyen Âge, le Midi portugais connaît une multiplication considérable du nombre de lieux politiquement et administrativement identifiés (qui correspond, mais en partie seulement, à la multiplication des habitats groupés, par un relatif regroupement du peuplement et surtout par croissance démographique absolue). L'église locale, avec ou sans titre paroissial, joue un rôle considérable dans le processus identitaire des communautés. La fondation des églises est du domaine du taisible ; rare est la documentation l'éclairant directement ${ }^{24}$; cependant, il ne fait guère de doute que l'église locale soit un élément essentiel d'affirmation locale et donc de multiplication des communautés : dans les «visitations" des commanderies, au Bas Moyen Âge, les lieux d'habitat sont toujours identifiés à travers leur temple et celuici est toujours visité en premier, même quand il n'est pas la source de revenus.

23. C'est le premier aspect qui l'emporte, à la fin du XIV siècle et au début du XV $\mathrm{XV}^{e}$, dans la pétition des conseils municipaux de Portalegre et Abrantes pour maintenir la participation des hommes de villages qui sont sous leur dépendance administrative (Assumar et Sardoal) à la procession du Corpus Christi se tenant au chef-lieu. Cf. Chancelarias portuguesas, op. cit., vol. I-2, p. 260, et M. S. AlvEs CONDE, Uma paisagem humanizada. O Médio Tejo nos finais da ldade Média, Cascais, 2000, t. I, p. 109 ; mais il faudrait de plus nombreuses occurrences pour trancher.

24. Il existe pourtant des méthodes bien éprouvées pour établir une chronologie, au moins grossière, de la constitution des réseaux, en se référant au nom des titulaires célestes - qui obéissent à des modes à peu près datées - et à la forme des territoires paroissiaux. Dans notre zone d'étude, ces méthodes sont difficiles à mettre en œuvre car la formation du réseau ecclésiastique, y compris dans sa dimension territoriale, s'opère sur un temps beaucoup plus bref (un ou deux siècles) que dans les régions où la christianisation est continue depuis les temps paléochrétiens; si les méthodes susmentionnées restent néanmoins opératoires, elles perdent leur principal intérêt, qui est chronologique. 
Un premier caractère doit être souligné d'emblée ; si l'acquisition de la paroissialité requiert des hommes de gros efforts et met en jeu un subtil rapport de pouvoirs, la détention d'un lieu de culte avec une desserte plus ou moins permanente rencontre peu d'opposition de la part des autorités, qui souscrivent à de pieuses considérations (le service de Dieu et le salut des âmes) d'autant plus volontiers que les usagers assurent la plus grande part des frais et ne remettent pas en cause la ponction seigneuriale des revenus ecclésiastiques ${ }^{25}$. Dès les débuts de la colonisation, de petits hameaux agricoles, collectifs ou constitués par un simple domaine foncier, sont pourvus de chapelles; leur mention est souvent générique - puisque ces chapelles, dépendant d'une église-mère, sont généralement évoquées dans des partages de revenus ecclésiastiques, qui ne citent que l'église centralisant les revenus - mais leur présence est attestée ponctuellement, par exemple autour de Santarém ${ }^{26}$. A la fin de notre période d'étude, en 1488 , le règlement général des visitations de l'ordre du Christ prévoit d'adapter systématiquement le nombre d'églises au volume des fidèles et à la répartition de leur habitat ${ }^{27}$. La construction d'une fortification est un investissement considérable et solidement encadré par l'autorité publique, donc souvent inaccessible, mais l'érection d'un lieu de culte est aisée à quiconque disposant de quelques moyens ${ }^{28}$ : les visitations de la fin du Moyen Âge mettent en scène des chapelles d'une grande simplicité architecturale et il est vraisemblable que les églises paroissiales primitives aient été aussi simples ${ }^{29}$, jusqu'à ce que la fierté et la ferveur des seigneurs et

25. Il est rare en tout cas que les seigneurs acceptent spontanément de sacrifier leurs revenus ou ceux d'une église décimale antérieure pour financer une nouvelle chapelle publique, comme c'est le cas en 1480, quand les visiteurs de l'ordre de Santiago complètent les revenus défaillants de l'église Saint-Sauveur de Sines aux dépens du prieur de Santiago do Cacém et en prélevant sur les revenus des reguengos de l'Ordre : éd. du texte de la "visitation " par L. A. DA FonseCA, "Vasco da Gama e a Ordem de Santiago " dans Ordens militares. Guerra, religião, poder e cultura. Actas do III encontro sobre Ordens Militares (Palmela. 22 a 25 de janeiro de 1998), I. C. F. FERNANDES coord., Lisbonne, 1999, t. II, p. 285-292.

26. Dans cette zone, sur la rivière Alviela, un ensemble de moulins signalé en 1206 est pourvu d'une chapelle dès 1220, pour desservir une population qui ne peut être très nombreuse (Arquivo do Cabido da Sé de Evora, CEC 3-III, fol. 4-5v).

27. M. Da Silva Castelo Branco, «Visitaçðes na Ordem de Cristo até finais do século XVI » dans As Ordens militares em Portugal e no Sul da Europa. Actas do II Encontro sobre Ordens militares (Palmela, 2, 3 e 4 de outubro de 1992), I. C. F. FERNANDES coord., Lisbonne, 1997, p. 407-430, voir p. 426.

28. La vita de Saint Martin de Soure met en scène la construction d'une nouvelle église dans le castrum de Soure dans les années 1120 , sur un site qui ne semble abriter qu'un petit groupe de colons : Hagiografia de Santa Cruz de Coimbra. Vida de D. Telo, vida de D. Teotónio, vida de Martinho de Soure, A. A. NASCIMENTo éd. et trad., Lisbonne, 1998, p. 235. Dans les années 1490, c'est un simple salarié étranger au concelho qui reconstruit une chapelle Saint-Pierre dans le territoire de Mértola: As comendas de Mértola e Alcaria Ruiva. As visitações e tombos da Ordem de Santiago 1482-1607, M. F. ROMBOUTS DE BARROS éd., Mértola, 1996, p. 97.

29. On notera que, malgré les ambiguiités de son vocabulaire, le plus ancien document méridional relatif aux églises (la donation des églises de Coina en 1184) est une transmission du seul ius fundi portant sur un patrimoine immobilier qui ne doit pas être de grande valeur. 
des fidèles suscitent parfois, dans les villages populeux, des reconstructions gothiques, elles-mêmes effacées par les splendeurs classiques et baroques (financées jusque dans les plus petits villages par l'or d'outre-mer). Quand les fondateurs sont des individus laïcs, il arrive souvent que l'entretien du bâtiment ne soit plus assuré au bout d'un temps assez bref, tombant alors dans l'abandon ou sous la tutelle publique, et il est donc aisé aux plus petites communautés de hameaux inorganiques de récupérer un lieu de culte sans même avoir à réaliser l'investissement de départ ${ }^{30}$. Il ne peut donc exister aucun rapport systématique et déterminant entre la taille d'une communauté et la présence d'un lieu de culte collectif.

L'église est un instrument d'autonomie mal connu mais essentiel ${ }^{31}$. Dans ce contexte, il ne faut jamais sous-estimer l'esprit de clocher des habitants d'un lieu, qui ont certainement une vision idéologique et morale de l'identité religieuse communautaire (comme élément de fierté et symbole de dignité collective) et qui appuient donc les démarches en ce sens, en dépit de toutes les contraintes qu'elle peut impliquer. Comme toujours, ce sont les éléments symboliques qui attestent le plus fortement la dimension idéologique de ce processus social : de la même manière que le village de Borba célèbre son indépendance politique par une inscription lapidaire, la prise en main d'un lieu de culte propre par les habitants d'Aldeia dos Crespos, près de Mértola, est un événement fondateur qui marque suffisamment les mémoires pour qu'on se souvienne de l'année précise un demi-siècle plus tard ${ }^{32}$; il s'agit dans le premier cas d'un habitat important et organique accédant au statut politique suprême (municipal) alors que les 39 foyers dispersés d'Aldeia dos Crespos obtiennent seulement un instrument communautaire, mais, à chaque niveau, il s'agit d'un processus identitaire majeur.

30. On observe cette situation à travers des documents tardifs, les visitations des ordres militaires, mais le mécanisme qu'elle révèle peut être très antérieur.

31. Pour cerner le rôle identitaire des églises, à côté d'une immense bibliographie proprement médiévistique, les schémas élaborés par les sociologues et anthropologues à propos du totémisme me semblent très utiles, notamment ce que dit Durkheim dans Les formes élémentaires de la vie religieuse sur le rôle fédérateur de l'objet religieux (dans notre cas un temple), transposant le lien social sur le plan du sacré et matérialisant la force surnaturelle (en l'occurrence celle du saint patron) pour en faire un objet de vénération. Tout en acceptant la fonction prioritairement sociale de l'objet sacré, $\mathrm{Cl}$. Lévi-Strauss réoriente ce schéma en faisant de l'identification d'un groupe à un objet sacré un système classificatoire qui permet à ce groupe de s'opposer aux autres (voyant donc l'identification surtout comme moyen de différenciation); c'est ce dernier phénomène que j'ai suggéré dans S. BoISSELLIER. "Organisation sociale et altérité culturelle dans l'hagionymie médiévale du Midi portugais ", Atalaya. Revue française d'études médiévales hispaniques, 11 (2000-2001), [Translatio hispanica. Traductions, transformations et transpositions textuelles en péninsule lbérique $X I I T^{\prime}-X V^{*}$ s. Hommage à Michel Garcia (Toulouse, 30 novembre 2001)], p. 141-181.

32. Cette chapelle du Saint-Esprit, fondée par des particuliers, averia ora cinquoenta e dous annos pouco mais ou menos que a dita irmida fora feita e edificada de novo pollos moradores $d$ ahi d arredor daquelle lemite (As comendas de Mértola e Alcaria Ruiva. op. cit., p. 92); c'est à partir de cette date de 1463 qu'y est desservi un culte collectif permanent et assumé par la communauté. 
Le système municipal introduit une forte originalité dans les rapports entre église et institutions financières locales, tant les communautés organiques sont souvent supposées être sorties de la fabrique (même dans les pays de consulat) pour l'aire française. Ici, les municipes des grandes communautés n'ont pas besoin de s'emparer de la fabrique pour construire d'abord leur existence même puis leur organisation financière car celle-là et celle-ci préexistent souvent à l'intégration de l'église dans le système paroissial (c'est-à-dire dans le système qui implique une organisation fiscale collective); au moins n'a-t-on pas de traces anciennes de ce processus. Sur ces chefs-lieux coloniaux anciens, on a vu que l'emprise seigneuriale s'exprime aussi à travers la monopolisation du droit de patronage et le concilium existe donc "à côté " de l'église décimale plutôt qu'en relation avec elle ; l'implication des communautés dans la fabrique n'est attestée que tardivement et on connaît fort mal la gestion financière des églises; il est cependant possible que les chapelles fondées par des membres de l'élite municipale finissent par être intégrées dans la gestion urbaine (sans lui donner naissance), surtout quand ces élites utilisent les finances urbaines pour servir leurs propres intérêts.

En revanche, dans les hameaux durablement dépendants, où l'église est utilisée comme instrument d'émancipation politique, la fabrique sert à organiser la communauté locale et donc à l'identifier par rapport à la juridiction englobante : si les chapelles sans habitat associé dans le termo municipal sont souvent sous la juridiction du concelho du chef-lieu ${ }^{33}$ et/ou sous le patronage direct du seigneur du municipe, les chapelles en rapport avec un hameau sont prises en main financièrement si ce n'est fondées par les habitants du lieu. Certes, les documents les plus précis restent assez vagues quant à cette organisation financière, se contentant souvent de signaler que le chapelain et l'entretien du bâtiment sont "payés par les habitants dudit lieu ", mais quelques mentions attestent qu'il existe un véritable budget, avec répartition des cotisations et même affectation de revenus publics. Dans ce cas, "la fonction crée l'organe " et la communauté, avec ce conseil de fabrique, devient institutionnelle ${ }^{34}$. En outre, dans le domaine purement ecclésiologique, l'existence d'une fabrique confère à ses membres le titre de paroissiens, alors que la fabrique et la nomination du chapelain semblent complètement indépendants des revenus canoniques du chef-lieu de paroisse et de la juridiction du recteur paroissial ; l'organisation financière prépare donc la paroissialité.

33. C'est le cas de plusieurs chapelles gérées par les chefs-lieux de Casével, Alvalade et Aljustrel à la fin du $X^{e}$ siècle : Documentos para a história da arte em Portugal 7. Visitações de Alvalade, Casével, Aljustrel e Setúbal, V. PAVÃo dos SANTOS éd., Lisbonne, 1969, p. 9-11, 11-12, 45 et 72-73.

34. Depuis 1463, les revenus d'un troupeau de moutons collectif sont affectés à la fabrique de la chapelle du Saint-Esprit d'Aldeia dos Crespos; en 1515, la maturation financière est suffisante pour que chaque village autour de Mértola se voie imposer par le seigneur (l'ordre de Santiago) l'institution d'un " bonhomme" du lieu comme juge et d'un notaire ( $A s$ comendas de Mértola e Alcaria Ruiva, op. cit., p. 92-93). 
L'église est aussi le support d'un saint patron communautaire. Bien que les dévotions se soient diffusées à l'origine dans le cadre d'une élite culturelle romaine constituant une internationale, le culte populaire des saints est fortement lié, dans le monde chrétien, aux communautés locales. P. David souligne ce processus d'appropriation des patrons célestes par les gens du cru, en forgeant des légendes qui font du saint un autochtone ou au moins un intervenant dans la localite ${ }^{35}$ : le sacré est donc fortement enraciné dans la topographie, c'est-à-dire dans un grand nombre de petits espaces différenciés, principalement à cause de la fonction de protection conférée aux saints ; dans les régions de peuplement concentré ou, au moins, de forte polarisation de l'habitat, la protection contre des fléaux globaux est conçue avant tout au niveau de la communauté d'habitants. Certes, il existe des saints patrons d'activité professionnelle (tardivement avec le développement des métiers et des confréries), de nations ou même de statuts sociaux mais le lien le plus organique est celui qui attache un culte et une communauté locale (au sens premier c'est-à-dire un groupe lié par la co-résidence). Le culte constitue donc un élément important de la spatialisation et de la territorialisation des groupes humains; une des manifestations les plus achevées est la procession suivant les limites du territoire municipal ou, sous d'autres cieux, le déplacement des villageois aux assemblées de paix derrière la bannière paroissiale. Le choix d'un patron est donc un élément important du processus identitaire collectif ; même si son fondement n'est pas le même et ses fonctions multiples, la paroisse constitue un bon instrument d'institutionnalisation du facteur religieux de l'esprit communautaire.

L'analyse des titres des églises majeures $m$ 'a conduit à y voir une recherche de consensus entre les habitants de l'ensemble du municipe par le choix de dévotions universelles, dont le monopole ne s'explique pas uniquement par les modes en cours ${ }^{36}$. Mais l'autonomisme des villages dépendants apparaît dans le choix de titres divergents; certes, les intercesseurs populaires, tels Sébastien, Roch, Blaise et Catherine, appliqués à des fondations de chapelles privées à l'origine, reflètent surtout l'opposition entre piété individuelle et piété collective, recouvrant partiellement une opposition entre la piété populaire et la piété institutionnalisée des églises-mères (quoique cette dernière soit assez peu influencée par les cultes liturgiques dans la région). Mais on trouve aussi les mêmes titres universels (Marie, Pierre, Jean, Jacques, Michel, Barthélemy) dans les églises majeures, c'est-à-dire des titres qui ont une fonction collective tout en différant du (ou des) vocable(s) des chefs-lieux de paroisse. Comme sur le plan politique, c'est donc la divergence qui l'emporte finalement sur l'intégration - sur un plan purement social et non pas spirituel car il est évident qu'un titre propre n'implique pas une hostilité

35. P. DAVID, « Le sanctoral hispanique et les patrons d'églises entre le Minho et le Mondego du IX ${ }^{e}$ au XII ${ }^{e}$ siècle ", dans ID., Études historiques sur la Galice et le Portugal du VF au XIF siècle, Lisbonne-Paris, 1947, p. 185-256.

36. S. BOISSELLIER, op. cit. 
envers le saint du chef-lieu en tant que tel mais symbolise seulement une spécificité. Puisque certaines de ces chapelles ont d'abord été fondées dans le cadre de domaines fonciers ou peut-être comme chapelles isolées ponctuant l'espace communautaire, on pourrait arguer que ces derniers titres, comme les intercesseurs, ont été choisis par des citoyens du chef-lieu et servent une politique d'intégration plutôt que d'identification; mais la fragilité des bâtiments auxquels sont attachés ces vocables, dans le cas des chapelles, impliquent des reconstructions souvent totales qui autorisent canoniquement les changements de titulature et les communautés naissantes des hameaux dépendants peuvent donc adopter une invocation céleste de leur choix quand elles prennent en main une de ces chapelles.

Quand on quitte les grands sites pour les hameaux (ou anciens hameaux ayant grossi), le rôle des lieux de culte apparaît plus nettement dans le processus d'identification. Dans la documentation écrite, c'est évidemment à travers les toponymes que cette influence se perçoit le mieux : le nom d'un lieu est fortement significatif non seulement de sa morphogenèse mais aussi de son intégration dans la trame du peuplement. En effet, le processus d'identification d'un lieu (i.e. d'une communauté) n'est pas endogène mais relatif car l'identité, matérielle et juridictionnelle, est une relation avec un environnement. La place accordée aux patronages célestes est notable parmi les toponymes des habitats secondaires; la présence d'une chapelle est donc un élément important et souvent capital. En effet, de nombreux lieux portant un hagiotoponyme ont un contenu, institutionnel et matériel, autre que leur rapport avec l'église et souvent antérieur à celui-ci (quoiqu'il soit difficile de fixer la chronologie): ainsi, des lieux ayant un contenu juridictionnel aussi fortement marqué que les reguengos ou une identité topographique et économique peuvent prendre comme nom définitif (mais souvent précoce) celui de la chapelle ${ }^{37}$. Même s'il n'est pas le point d'ancrage matériel de l'agglomération, le lieu de culte est l'étape essentielle du processus identitaire des hameaux groupés.

Mais l'église (au sens large) est fondatrice de l'identité même quand elle se situe au milieu d'une zone de résidences dispersées. Non seulement elle constitue un "centre" de rassemblement physique conférant une polarité à une zone qui serait autrement sans limites, ce qui permet à ses alentours de n'être pas seulement un morceau indifférencié du termo d'un chef-lieu ${ }^{38}$, mais elle est aussi le seul élément à valeur juridique (encadré par une autorité et s'intégrant dans une administration d'ensemble): ses

37. Ainsi, l'ensemble de domaines agricoles formant le lieu-dit Caia (nom de la rivière qui le traverse) au début du XV $\mathrm{XV}^{e}$ siècle, qui finit par prendre le nom de Caia e São Pedro ou le reguengo (qui a une matérialité bien supérieure à un simple domaine inclus dans un terroir) proche de Punhete qui s'appelle, au moins dès la fin du XIV ${ }^{\mathrm{e}}$ siècle, Santa Margarida (Chancelarias portuguesas, op. cit., vol. I/1, p. 248-249).

38. En 1482, ce sont les chapelles de plusicurs villages des alentours de Mértola qui semblent définir les habitats, plus qu'une éventuelle massivité morphologique, car leurs habitants sont qualifiés par leur " appartenance " à ces chapelles : dezer missa [...] nas ssobredictas irmidas aos comarcãos delas (As comendas de Mértola e Alcaria Ruiva, op. cit., p. 60). 
«paroissiens » peuvent donc être définis autrement que par leur inclusion (en situation de dépendance) dans un territoire politique (municipal). Dans le domaine toponymique, on peut opposer les deux habitats satellites de Mértola, Corte do Pinto et le futur Santana de Cambas : dans le premier, le contenu originel (un domaine portant un éponyme) persiste dans le nom parce qu'une petite agglomération a été cristallisée par le centre d'exploitation et parce que le cadre foncier (droit de propriété d'un seul détenteur) est fondateur; dans le second, la dispersion est telle que l'habitat n'est pas encore nettement identifié en 1515 et c'est seulement la chapelle qui peut imposer son nom comme élément fondateur d'identitéc ${ }^{39}$. Il est possible que les autorités intervenant dans l'organisation des lieux de culte prennent en compte la dispersion du peuplement comme un facteur favorable à la fondation (si ce n'est plus) de chapelles moins "rentables" administrativement (desservant moins de fidèles) que les temples des habitats groupés ${ }^{40}$.

En guise de conclusion, soulignons que, aux échelles supra-locale et régionale de l'organisation spatiale, les églises locales fondent la domination de l'espace par création de territoires. Longtemps avant la délimitation des territoires paroissiaux, qui est rarement antérieure au milieu du XIII ${ }^{\mathrm{e}}$ siècle (sauf dans les tissus urbains pluri-paroissiaux), les décimaires constituent les plus anciens territoires concrets; beaucoup plus que dans le cadre des territoires politiques municipaux (termo) bien plus vastes et englobant de nombreux habitats, c'est à ce niveau local, celui du village, que se concrétisent les éléments d'organisation communautaire. Sans que l'église qui les polarise n'acquière forcément le titre paroissial, ces territoires forment la cellule territoriale de base, qui appuie l'autonomie politique de nombreux villages modestes (promus au rang de vilas dans les années 13201520).

39. La chapelle est identifiée par son vocable et localisée seulement comme appartenant au territoire de Mértola. Puis le lieu de Cambas est mentionné plus loin à propos d'un problème agraire, avec mention incidente de la chapelle (ibid., p. 87 et 119-120). Le lieu n'est donc pas créé mais identifié par la chapelle, qui est probablement périphérique par rapport aux résidences.

40. En 1488, on l'a vu, le règlement des visitations de l'ordre du Christ prend en compte non seulement le nombre d'habitants mais aussi la dispersion de l'habitat dans la fondation de nouvelles églises se alguma comenda achardes de tamanho termo e fregueses tam espalhados que vos nam pareça rrezam de a huuma igreja soo averem de viir [...] hordenarees de se fazer e poer se nella cura (M. DA SILVA CASTELO BRANCO, op. cit., p. 426). 